Bull. Korean Math. Soc. 46 (2009), No. 6, pp. 1079-1089

DOI 10.4134/BKMS.2009.46.6.1079

\title{
UNIQUENESS THEOREMS OF MEROMORPHIC FUNCTIONS OF A CERTAIN FORM
}

\author{
Junfeng Xu, Qi HAN, AND JilOng Zhang
}

\begin{abstract}
In this paper, we shall show that for any entire function $f$, the function of the form $f^{m}\left(f^{n}-1\right) f^{\prime}$ has no non-zero finite Picard value for all positive integers $m, n \in \mathbb{N}$ possibly except for the special case $m=n=1$. Furthermore, we shall also show that for any two nonconstant meromorphic functions $f$ and $g$, if $f^{m}\left(f^{n}-1\right) f^{\prime}$ and $g^{m}\left(g^{n}-1\right) g^{\prime}$ share the value 1 weakly, then $f \equiv g$ provided that $m$ and $n$ satisfy some conditions. In particular, if $f$ and $g$ are entire, then the restrictions on $m$ and $n$ could be greatly reduced.
\end{abstract}

\section{Introduction and main results}

In this paper, a meromorphic function will always mean meromorphic in the complex plane $\mathbb{C}$. We adopt the standard notations in the Nevanlinna value distribution theory of meromorphic functions such as $T(r, f), m(r, f), N(r, f)$ and $\bar{N}(r, f)$ as explained in $[4,7,12]$. For any non-constant meromorphic function $f$, we denote by $S(r, f)$ any quantity satisfying $S(r, f)=o(T(r, f))$, possibly outside a set of finite linear measure that is not necessarily the same at each occurrence.

Let $f$ be a non-constant meromorphic function on $\mathbb{C}$, let $a \in \mathbb{C}$ be a finite value, and let $k$ be a positive integer or infinity. We denote by $E(a, f)$ the set of zeros of $f-a$ and count multiplicities, while by $\bar{E}(a, f)$ the set of zeros of $f-a$ but ignore multiplicities. Also, we denote by $E_{k}(a, f)$ the set of zeros of $f-a$ with multiplicities less than or equal to $k$ and count multiplicities. Obviously, $E(a, f)=E_{\infty)}(a, f)$. For the value $\infty$, define $E(\infty, f):=E(0,1 / f)$. $\bar{E}(\infty, f)$ and $E_{k)}(\infty, f)$ are similarly defined. For $a \in \mathbb{C} \cup\{\infty\}$, we denote by $N_{k)}(r, 1 /(f-a))$ the counting function corresponding to the set $E_{k)}(a, f)$, while by $N_{(k+1}(r, 1 /(f-a))$ the counting function corresponding to the set

Received September 1, 2008.

2000 Mathematics Subject Classification. 30D35, 30D20, 30D30.

Key words and phrases. entire function, meromorphic function, Picard value.

Supported by the NSF of China (10771121), NSF of Guangdong Province (9452902001003278) and Excellent Young Fund of Department of Education of Guangdong (LYM08097). 
$E_{(k+1}(a, f):=E(a, f) \backslash E_{k)}(a, f)$. Also, we denote by $\bar{N}_{k)}(r, 1 /(f-a))$ and $\bar{N}_{(k+1}(r, 1 /(f-a))$ the reduced forms of $N_{k)}(r, 1 /(f-a))$ and $N_{(k+1}(r, 1 /(f-$ $a)$ ), respectively.

Hayman proposed the well-known conjecture in [5].

Hayman Conjecture. If an entire function $f$ satisfies $f^{n} f^{\prime} \neq 1$ for all $n \in \mathbb{N}$, then $f$ is a constant.

In fact, it has been affirmed by Hayman himself in [6] for the cases $n>1$ while by Clunie in [2] for the cases $n \geq 1$, respectively. In 1997, C. C. Yang and $\mathrm{X}$. H. Hua studied the unicity of the differential monomials $f^{n} f^{\prime}$ and proved the following uniqueness theorem in [10].

Theorem A. Let $f$ and $g$ be two non-constant meromorphic functions, let $a$ be a non-zero finite value, and let $n \geq 11$, be a positive integer. If $f^{n} f^{\prime}$ and $g^{n} g^{\prime}$ share a $C M$, then either $f=\bar{d} g$ for some $(n+1)$-th root of unity $d$, or $f=c_{1} e^{c z}$ and $g=c_{2} e^{-c z}$ for three non-zero constants $c, c_{1}$ and $c_{2}$ with $\left(c_{1} c_{2}\right)^{n+1} c^{2}=-a^{2}$.

In 2001, by using the same argument as that in [6], M. L. Fang and W. Hong studied the value distribution of $f^{m}(f-1) f^{\prime}$ with an entire function $f$ and proved the following Theorem B. Also, they discussed the uniqueness problem of $f^{m}(f-1) f^{\prime}$ with an entire function $f$ and obtained the following Theorem $\mathrm{C}$ (see [3]).

Theorem B. If an entire function $f$ satisfies $f^{m}(f-1) f^{\prime} \neq 1$ for all $m \in \mathbb{N}$ with $m \geq 2$, then $f$ is a constant.

Theorem C. Let $f$ and $g$ be two non-constant entire functions. If $f^{m}(f-1) f^{\prime}$ and $g^{m}(g-1) g^{\prime}$ share the value $1 C M$, then $f \equiv g$ provided that $m \geq 11$.

In 2004, W. C. Lin and H. X. Yi improved Theorem C, reducing the restriction on the lower bound of the positive integer $m$ from 11 to 7 (see [8]). Furthermore, in that same paper, they studied the uniqueness problem of meromorphic functions with the same form as that shown above and obtained the following result.

Theorem D. Let $f$ and $g$ be two non-constant meromorphic functions. If $f^{m}(f-1) f^{\prime}$ and $g^{m}(g-1) g^{\prime}$ share the value $1 C M$, and if $\Theta(\infty, f)>\frac{2}{m+1}$, then $f \equiv g$ provided that $m \geq 11$. Where $\Theta(\infty, f):=1-\limsup _{r \rightarrow \infty} \frac{\bar{N}(r, f)}{T(r, f)}$.

In this paper, we shall consider the function with the form $f^{m}\left(f^{n}-1\right) f^{\prime}$ and prove the following uniqueness theorems.

Theorem 1.1. Let $f$ be a non-constant entire function. Then, $f^{m}\left(f^{n}-1\right) f^{\prime}$ has no non-zero finite Picard value for all positive integers $m, n \in \mathbb{N}$ possibly except for the special case $m=n=1$. 
Theorem 1.2. Let $f$ and $g$ be two non-constant meromorphic functions. If $E_{3)}\left(1, f^{m}\left(f^{n}-1\right) f^{\prime}\right)=E_{3)}\left(1, g^{m}\left(g^{n}-1\right) g^{\prime}\right)$, then $f \equiv g$ provided that $m>$ $n+10, n \geq 2$ and $(m+1, n)=1$.

Theorem 1.3. Let $f$ and $g$ be two non-constant entire functions. If

$$
E_{3)}\left(1, f^{m}\left(f^{n}-1\right) f^{\prime}\right)=E_{3)}\left(1, g^{m}\left(g^{n}-1\right) g^{\prime}\right),
$$

then $f \equiv g$ provided that $m>n+5$.

Theorem 1.4. Let $f$ and $g$ be two non-constant meromorphic functions. If $E_{2)}\left(1, f^{m}\left(f^{n}-1\right) f^{\prime}\right)=E_{2)}\left(1, g^{m}\left(g^{n}-1\right) g^{\prime}\right)$, then $f \equiv g$ provided that $m>$ $\frac{3 n}{2}+12, n \geq 2$ and $(m+1, n)=1$.

Theorem 1.5. Let $f$ and $g$ be two non-constant entire functions. If

$$
E_{2)}\left(1, f^{m}\left(f^{n}-1\right) f^{\prime}\right)=E_{2)}\left(1, g^{m}\left(g^{n}-1\right) g^{\prime}\right) \text {, }
$$

then $f \equiv g$ provided that $m>\frac{3 n+13}{2}$.

Theorem 1.6. Let $f$ and $g$ be two non-constant meromorphic functions. If $E_{1)}\left(1, f^{m}\left(f^{n}-1\right) f^{\prime}\right)=E_{1)}\left(1, g^{m}\left(g^{n}-1\right) g^{\prime}\right)$, then $f \equiv g$ provided that $m>$ $3 n+18, n \geq 2$ and $(m+1, n)=1$.

Theorem 1.7. Let $f$ and $g$ be two non-constant entire functions. If

$$
E_{1)}\left(1, f^{m}\left(f^{n}-1\right) f^{\prime}\right)=E_{1)}\left(1, g^{m}\left(g^{n}-1\right) g^{\prime}\right),
$$

then $f \equiv g$ provided that $m>3 n+11$.

Remark 1.8. Obviously, Theorem 1.1 is an improvement of Theorem B while Theorem 1.3 is an improvement of Theorem $\mathrm{C}$ and Theorem 1 in [8].

Example 1. Set $f:=\frac{e^{z}}{e^{z}-1}$ and $g:=\zeta f=\frac{\zeta e^{z}}{e^{z}-1}$ for some primitive $n$-th root of unity $\zeta$ with $\zeta \neq 1$ and $n \geq 2$. Then, for arbitrary positive integer $m \in \mathbb{N}$,

$$
f^{m}\left(f^{n}-1\right) f^{\prime}=-\frac{e^{(m+1) z}\left(e^{n z}-\left(e^{z}-1\right)^{n}\right)}{\left(e^{z}-1\right)^{m+n+2}}
$$

and

$$
g^{m}\left(g^{n}-1\right) g^{\prime}=-\frac{\zeta^{m+1} e^{(m+1) z}\left(e^{n z}-\left(e^{z}-1\right)^{n}\right)}{\left(e^{z}-1\right)^{m+n+2}} .
$$

Hence, $f^{m}\left(f^{n}-1\right) f^{\prime}$ and $g^{m}\left(g^{n}-1\right) g^{\prime}$ share the value 0 CM. However, $f \not \equiv g$.

Example 2. Set $f:=e^{z}$ and $g:=\zeta f=\zeta e^{z}$ for some primitive $n$-th root of unity $\zeta$ with $\zeta \neq 1$ and $n \geq 2$. Then, for arbitrary positive integer $m \in \mathbb{N}$,

$$
f^{m}\left(f^{n}-1\right) f^{\prime}=e^{(m+1) z}\left(e^{n z}-1\right), \quad g^{m}\left(g^{n}-1\right) g^{\prime}=\zeta^{m+1} e^{(m+1) z}\left(e^{n z}-1\right) .
$$

Hence, $f^{m}\left(f^{n}-1\right) f^{\prime}$ and $g^{m}\left(g^{n}-1\right) g^{\prime}$ share the value 0 CM. However, $f \not \equiv g$.

Example 3. Set $f:=e^{z}$ and $g:=e^{-z}$. Then, for arbitrary positive integers $m, n \in \mathbb{N}$,

$$
f^{m}\left(f^{n}-1\right) f^{\prime}=e^{(m+1) z}\left(e^{n z}-1\right), \quad g^{m}\left(g^{n}-1\right) g^{\prime}=e^{-(m+n+1) z}\left(e^{n z}-1\right) .
$$

Hence, $f^{m}\left(f^{n}-1\right) f^{\prime}$ and $g^{m}\left(g^{n}-1\right) g^{\prime}$ share the value 0 CM. However, $f \not \equiv g$. 
Example 4. Set $f:=e^{z}+\frac{1}{2}$ and $g:=-e^{z}+\frac{1}{2}$. Then, for $m=n=1$,

$$
f(f-1) f^{\prime}=e^{z}\left(e^{z}+\frac{1}{2}\right)\left(e^{z}-\frac{1}{2}\right), \quad g(g-1) g^{\prime}=-e^{z}\left(e^{z}+\frac{1}{2}\right)\left(e^{z}-\frac{1}{2}\right) .
$$

Hence, $f(f-1) f^{\prime}$ and $g(g-1) g^{\prime}$ share the value 0 CM. However, $f \not \equiv g$.

\section{Some lemmas}

Lemma 2.1. Let $f$ and $g$ be two non-constant meromorphic functions satisfying $E_{k)}(1, f)=E_{k)}(1, g)$ for some positive integer $k \in \mathbb{N}$. Define $H$ as below

$$
H=\left(\frac{f^{\prime \prime}}{f^{\prime}}-2 \frac{f^{\prime}}{f-1}\right)-\left(\frac{g^{\prime \prime}}{g^{\prime}}-2 \frac{g^{\prime}}{g-1}\right) .
$$

If $H \not \equiv 0$, then

$$
\begin{aligned}
N(r, H) \leq & \bar{N}_{(2}(r, f)+\bar{N}_{(2}\left(r, \frac{1}{f}\right)+\bar{N}_{(2}(r, g)+\bar{N}_{(2}\left(r, \frac{1}{g}\right)+N_{0}\left(r, \frac{1}{f^{\prime}}\right)+N_{0}\left(r, \frac{1}{g^{\prime}}\right) \\
& +\bar{N}_{(k+1}\left(r, \frac{1}{f-1}\right)+\bar{N}_{(k+1}\left(r, \frac{1}{g-1}\right)+S(r, f)+S(r, g),
\end{aligned}
$$

where $N_{0}\left(r, \frac{1}{f^{\prime}}\right)$ denotes the counting function of zeros of $f^{\prime}$ but not zeros of $f(f-1)$, and $N_{0}\left(r, \frac{1}{g^{\prime}}\right)$ is similarly defined.

Proof. It is easy to see that simple poles of $f$ is not poles of $\frac{f^{\prime \prime}}{f^{\prime}}-\frac{2 f^{\prime}}{f-1}$ and simple poles of $g$ is not poles of $\frac{g^{\prime \prime}}{g^{\prime}}-\frac{2 g^{\prime}}{g-1}$. From the assumption that $E_{k)}(1, f)=$ $E_{k)}(1, g)$, we can easily obtain the conclusion.

Lemma 2.2 ([13]). Under the condition of Lemma 2.1, we have

$$
N_{1)}\left(r, \frac{1}{f-1}\right)=N_{1)}\left(r, \frac{1}{g-1}\right) \leq N(r, H)+S(r, f)+S(r, g) .
$$

Lemma 2.3 ([13]). Let $H$ be defined as above. If $H \equiv 0$, then either $f \equiv g$ or $f g \equiv 1$ provided that

$$
\limsup _{r \rightarrow \infty, r \in I} \frac{\bar{N}(r, f)+\bar{N}\left(r, \frac{1}{f}\right)+\bar{N}(r, g)+\bar{N}\left(r, \frac{1}{g}\right)}{T(r)}<1,
$$

where $T(r):=\max \{T(r, f), T(r, g)\}$ and $I$ is a set with infinite linear measure.

Lemma 2.4 ([11, 13]). Let $m$ and $n$ be two positive integers such that $m \geq 5$, $(m, n)=1$ and $1 \leq n \leq m-2$. For any two non-constant meromorphic functions $f$ and $g$, if $P(f) \equiv P(g)$, then $f \equiv g$. Where $P(z)=z^{m}+a z^{n}+b$, a polynomial, with $a \in \mathbb{C} \backslash\{0\}$ and $b \in \mathbb{C}$. 


\section{Proof of Theorem 1.1}

Since every polynomial has no finite Picard value, so without loss of generality, we may assume that $f$ is transcendental.

Define $F:=f^{m}\left(f^{n}-1\right) f^{\prime}$ and $F_{1}:=\frac{f^{m+n+1}}{m+n+1}-\frac{f^{m+1}}{m+1}$. Then, $F_{1}^{\prime}=F$.

At first, let's assume $m \geq 2$ and $F \neq a$ for some non-zero finite value $a$. Then, applying the second main theorem to $F$, together with the lemma of logarithmic derivative and Valirons' Lemma, to conclude that

$$
\begin{aligned}
& (m+n+1) T(r, f) \\
= & T\left(r, F_{1}\right)+O(1) \\
\leq & T(r, F)+N\left(r, \frac{1}{F_{1}}\right)-N\left(r, \frac{1}{F}\right)+S(r, f) \\
\leq & \bar{N}\left(r, \frac{1}{F}\right)+N\left(r, \frac{1}{F_{1}}\right)-N\left(r, \frac{1}{F}\right)+S(r, f) \\
\leq & \bar{N}\left(r, \frac{1}{f}\right)+\sum_{j=1}^{n} \bar{N}\left(r, \frac{1}{f-\omega_{j}}\right)+\bar{N}\left(r, \frac{1}{f^{\prime}}\right)+(m+1) N\left(r, \frac{1}{f}\right) \\
& +n T(r, f)-m N\left(r, \frac{1}{f}\right)-\sum_{j=1}^{n} N\left(r, \frac{1}{f-\omega_{j}}\right)-N\left(r, \frac{1}{f^{\prime}}\right)+S(r, f) \\
\leq & 2 N\left(r, \frac{1}{f}\right)+n T(r, f)+S(r, f) \leq(n+2) T(r, f)+S(r, f),
\end{aligned}
$$

where $\omega_{j}^{n}=1$, are the $n$-th roots of unit for $j=1,2, \ldots, n$. However, the above inequality means $(m-1) T(r, f) \leq S(r, f)$, which is possible since $m-1>0$.

Now, we consider the special case $m=1$.

If $n=2$, we define $\varphi=f^{2}-1$. Obviously, $f\left(f^{2}-1\right) f^{\prime}$ can be rewritten as $\frac{1}{2} \varphi \varphi^{\prime}$. Hence, it has no non-zero finite Picard value by Hayman Conjecture.

If $n \geq 3$, we proceed our proof by contradiction. Assume, to the contrary, that there exists a value $a \in \mathbb{C} \backslash\{0\}$ such that $F-a=p e^{\alpha}$. Then,

$$
f\left(f^{n}-1\right) f^{\prime}-a=p e^{\alpha}
$$

where $p$ is a non-zero polynomial, and $\alpha$ is a non-constant entire function satisfying $T\left(r, e^{\alpha}\right)=O(T(r, f))$.

Rewriting (3.1) as

$$
f^{n+1} f^{\prime}-f f^{\prime}-a=p e^{\alpha}
$$

and taking derivatives on both sides of $(3.2)$, we get

$$
(n+1) f^{n}\left(f^{\prime}\right)^{2}+f^{n+1} f^{\prime \prime}-\left(f^{\prime}\right)^{2}-f f^{\prime \prime}=\left(p^{\prime}+p \alpha^{\prime}\right) e^{\alpha} .
$$

Eliminating $e^{\alpha}$ by the above two equations yields

$$
f^{n}\left((n+1)\left(f^{\prime}\right)^{2}+f f^{\prime \prime}-\beta f f^{\prime}\right)=\left(f^{\prime}\right)^{2}+f f^{\prime \prime}-\beta f f^{\prime}-a \beta,
$$

where $\beta:=\left(\alpha^{\prime}+\frac{p^{\prime}}{p}\right)$ satisfying $T(r, \beta)=S(r, f)$. 
Applying Clunie's Lemma $([1,4])$ to $(3.4)$ for $n \geq 3$ and $\gamma_{Q[f]}=2$ to derive that $m(r, P[f])=S(r, f)$ and $m(r, f P[f])=S(r, f)$, where $P[f]:=$ $(n+1)\left(f^{\prime}\right)^{2}+f f^{\prime \prime}-\beta f f^{\prime}$ and $Q[f]:=\left(f^{\prime}\right)^{2}+f f^{\prime \prime}-\beta f f^{\prime}-a \beta$. If $P[f] \equiv 0$, then $Q[f] \equiv 0$, too. Thus we get $n\left(f^{\prime}\right)^{2}+a \beta \equiv 0$, which means $T\left(r, f^{\prime}\right)=S(r, f)$; then $T\left(r, f^{\prime \prime}\right)=S(r, f)$ by the lemma of logarithmic derivative. Since now $f \equiv \frac{(n+1)\left(f^{\prime}\right)^{2}}{\beta f^{\prime}-f^{\prime \prime}}$, then $T(r, f)=S(r, f)$, which is impossible. So $P[f] \not \equiv 0$. Since we assume $f$ is entire, then

$$
\begin{aligned}
T(r, f) & =T\left(r, \frac{f P[f]}{P[f]}\right)+O(1) \\
& \leq T(r, f P[f])+T(r, P[f])+O(1) \leq S(r, f) .
\end{aligned}
$$

This contradiction finishes the proof.

\section{Proof of Theorem 1.2}

Similar to the proof of Theorem 1.1, we get

$$
\begin{aligned}
(m+n+1) T(r, f) & =T\left(r, F_{1}\right)+O(1) \\
& \leq T(r, F)+N\left(r, \frac{1}{F_{1}}\right)-N\left(r, \frac{1}{F}\right)+S(r, f), \\
(m+n+1) T(r, g) & =T\left(r, G_{1}\right)+O(1) \\
& \leq T(r, G)+N\left(r, \frac{1}{G_{1}}\right)-N\left(r, \frac{1}{G}\right)+S(r, g),
\end{aligned}
$$

where $G$ and $G_{1}$ are similarly defined as that of $F$ and $F_{1}$ in Theorem 1.1.

First of all, we suppose that $H \not \equiv 0$, where we replace $f$ and $g$ by $F$ and $G$ respectively in Lemmas 2.1 and 2.2. Then,

$$
\begin{aligned}
N_{1)}\left(r, \frac{1}{F-1}\right) \leq & \bar{N}_{(2}(r, F)+\bar{N}_{(2}(r, G)+\bar{N}_{(2}\left(r, \frac{1}{F}\right)+\bar{N}_{(2}\left(r, \frac{1}{G}\right)+N_{0}\left(r, \frac{1}{F^{\prime}}\right) \\
& +N_{0}\left(r, \frac{1}{G^{\prime}}\right)+\bar{N}_{(4}\left(r, \frac{1}{F-1}\right)+\bar{N}_{(4}\left(r, \frac{1}{G-1}\right) \\
& +S(r, f)+S(r, g) .
\end{aligned}
$$

Applying the second main theorem to $F$ and $G$ jointly to obtain that

$$
\begin{aligned}
& T(r, F)+T(r, G) \\
\leq & \bar{N}\left(r, \frac{1}{F}\right)+\bar{N}\left(r, \frac{1}{F-1}\right)+\bar{N}(r, F)+\bar{N}\left(r, \frac{1}{G}\right)+\bar{N}\left(r, \frac{1}{G-1}\right) \\
& +\bar{N}(r, G)-N_{0}\left(r, \frac{1}{F^{\prime}}\right)-N_{0}\left(r, \frac{1}{G^{\prime}}\right)+S(r, f)+S(r, g) .
\end{aligned}
$$

Noting that

$$
\bar{N}\left(r, \frac{1}{F-1}\right)-\frac{1}{2} N_{1)}\left(r, \frac{1}{F-1}\right)+\bar{N}_{(4}\left(r, \frac{1}{F-1}\right) \leq \frac{1}{2} N\left(r, \frac{1}{F-1}\right) \leq \frac{1}{2} T(r, F),
$$


(4.6)

$$
\bar{N}\left(r, \frac{1}{G-1}\right)-\frac{1}{2} N_{1)}\left(r, \frac{1}{G-1}\right)+\bar{N}_{(4}\left(r, \frac{1}{G-1}\right) \leq \frac{1}{2} N\left(r, \frac{1}{G-1}\right) \leq \frac{1}{2} T(r, G) ;
$$

from (4.3)-(4.6), we have

$$
\begin{aligned}
T(r, F)+T(r, G) \leq & 2\left\{N_{2}(r, F)+N_{2}(r, G)+N_{2}\left(r, \frac{1}{F}\right)+N_{2}\left(r, \frac{1}{G}\right)\right\} \\
& +S(r, f)+S(r, g),
\end{aligned}
$$

where

$$
N_{2}(r, F):=\bar{N}(r, F)+N_{(2}(r, F), \quad N_{2}(r, 1 / F):=\bar{N}(r, 1 / F)+\bar{N}_{(2}(r, 1 / F),
$$

and $N_{2}(r, G)$ and $N_{2}(r, 1 / G)$ are similarly defined.

From the assumptions of Theorem 1.2, we get

(4.8) $N_{2}(r, F)+N_{2}\left(r, \frac{1}{F}\right) \leq 2 \bar{N}(r, f)+2 N\left(r, \frac{1}{f}\right)+\sum_{i=1}^{n} N\left(r, \frac{1}{f-\omega_{i}}\right)+N\left(r, \frac{1}{f^{\prime}}\right)$,

(4.9) $N_{2}(r, G)+N_{2}\left(r, \frac{1}{G}\right) \leq 2 \bar{N}(r, g)+2 N\left(r, \frac{1}{g}\right)+\sum_{i=1}^{n} N\left(r, \frac{1}{g-\omega_{i}}\right)+N\left(r, \frac{1}{g^{\prime}}\right)$.

Noting that

(4.10) $N\left(r, \frac{1}{F_{1}}\right)-N\left(r, \frac{1}{F}\right) \leq n T(r, f)+N\left(r, \frac{1}{f}\right)-\sum_{i=1}^{n} N\left(r, \frac{1}{f-\omega_{i}}\right)-N\left(r, \frac{1}{f^{\prime}}\right)$,

(4.11) $N\left(r, \frac{1}{G_{1}}\right)-N\left(r, \frac{1}{G}\right) \leq n T(r, g)+N\left(r, \frac{1}{g}\right)-\sum_{i=1}^{n} N\left(r, \frac{1}{g-\omega_{i}}\right)-N\left(r, \frac{1}{g^{\prime}}\right)$,

and

$$
N\left(r, \frac{1}{f^{\prime}}\right) \leq N\left(r, \frac{1}{f}\right)+\bar{N}(r, f)+S(r, f) ;
$$

from (4.1)-(4.2) and (4.7)-(4.12), we have

$$
(m+n+1)(T(r, f)+T(r, g)) \leq(2 n+11)(T(r, f)+T(r, g))+S(r, f)+S(r, g) .
$$

Then,

$$
(m-n-10)(T(r, f)+T(r, g)) \leq S(r, f)+S(r, g),
$$

which is impossible since we assume $m>n+10$.

Now we consider the case $H \equiv 0$. It is not difficult to see

$$
\frac{1}{G-1}=\frac{A}{F-1}+B
$$


for some constants $A \in \mathbb{C} \backslash\{0\}$ and $B \in \mathbb{C}$. Obviously,

$$
\begin{aligned}
& \bar{N}(r, F)+\bar{N}\left(r, \frac{1}{F}\right)+\bar{N}(r, G)+\bar{N}\left(r, \frac{1}{G}\right) \\
\leq & \bar{N}(r, f)+\bar{N}\left(r, \frac{1}{f}\right)+\sum_{i=1}^{n} \bar{N}\left(r, \frac{1}{f-\omega_{i}}\right)+\bar{N}\left(r, \frac{1}{f^{\prime}}\right)+\bar{N}(r, g) \\
& +\bar{N}\left(r, \frac{1}{g}\right)+\sum_{i=1}^{n} \bar{N}\left(r, \frac{1}{g-\omega_{i}}\right)+\bar{N}\left(r, \frac{1}{g^{\prime}}\right)+S(r, f)+S(r, g) \\
\leq & (2 n+4) T_{0}(r)+\bar{N}\left(r, \frac{1}{f^{\prime}}\right)+\bar{N}\left(r, \frac{1}{g^{\prime}}\right)+S(r, f)+S(r, g),
\end{aligned}
$$

where $T_{0}(r):=\max \{T(r, f), T(r, g)\}$.

Noting that

$$
\begin{aligned}
& T\left(r, f^{m}\left(f^{n}-1\right)\right) \\
& \leq m\left(r, f^{m}\left(f^{n}-1\right) f^{\prime}\right)+m\left(r, \frac{1}{f^{\prime}}\right)+N\left(r, f^{m}\left(f^{n}-1\right) f^{\prime}\right)+S(r, f) \\
& \leq T(r, F)+m\left(r, \frac{1}{f^{\prime}}\right)+S(r, f), \\
& N\left(r, \frac{1}{f^{\prime}}\right) \leq T\left(r, f^{\prime}\right)-m\left(r, \frac{1}{f^{\prime}}\right)+S(r, f) \leq 2 T(r, f)-m\left(r, \frac{1}{f^{\prime}}\right)+S(r, f),
\end{aligned}
$$

and $2 n+8<m+n$, from (4.13) we get

$$
\bar{N}(r, F)+\bar{N}\left(r, \frac{1}{F}\right)+\bar{N}(r, G)+\bar{N}\left(r, \frac{1}{G}\right)<T(r)+S(r, F)+S(r, G),
$$

where $T(r):=\max \{T(r, F), T(r, G)\}$.

Thus, by Lemma 2.3 , we have either $F G \equiv 1$ or $F \equiv G$.

Now we consider the following two cases.

Case (i): $F G \equiv 1$.

We have

$$
f^{m}\left(f^{n}-1\right) f^{\prime} g^{m}\left(g^{n}-1\right) g^{\prime} \equiv 1 .
$$

Let $z_{0}$ be a zero of $f-\omega_{i}$ with multiplicity $p$. Then it must be a pole of $g$, thus $2 p-1 \geq(m+n+1)+1$, which means $p \geq \frac{m+n+3}{2}$.

If $n \geq 3$, by the second main theorem, we have

$$
\begin{aligned}
T(r, f) & \leq \sum_{i=1}^{3} \bar{N}\left(r, \frac{1}{f-\omega_{i}}\right)+S(r, f) \\
& \leq \sum_{i=1}^{3} \frac{2}{m+n+3} N\left(r, \frac{1}{f-\omega_{i}}\right)+S(r, f) \\
& \leq \frac{6}{m+n+3} T(r, f)+S(r, f),
\end{aligned}
$$

which is absurd since we assume $m>n+10$. 
If $n=2$, we see that a zero $z_{1}$ of $f$ with multiplicity $q$ must be a pole of $g$ with multiplicity $q^{*}$ satisfying $m q+q-1=(m+2+1) q^{*}+1$. Thus, $(m+1)\left(q-q^{*}\right)=2 q^{*}+2$, which means $q \geq q^{*}+1 \geq \frac{m+1}{2}$. Similar as the cases $n \geq 3$, we get

$$
\begin{aligned}
T(r, f) & \leq \bar{N}\left(r, \frac{1}{f}\right)+\bar{N}\left(r, \frac{1}{f+1}\right)+\bar{N}\left(r, \frac{1}{f-1}\right)+S(r, f) \\
& \leq \frac{2}{m+1} N\left(r, \frac{1}{f}\right)+\frac{2}{m+5} N\left(r, \frac{1}{f+1}\right)+\frac{2}{m+5} N\left(r, \frac{1}{f-1}\right)+S(r, f) \\
& \leq \frac{2}{m+1} T(r, f)+\frac{4}{m+5} T(r, f)+S(r, f)
\end{aligned}
$$

which is absurd since we assume $m>n+10$.

Case (ii): $F \equiv G$.

We have

$$
F_{1} \equiv G_{1}+c \quad(c \in \mathbb{C})
$$

If $c \neq 0$, then we have

$$
\begin{aligned}
(m+n+1) T(r, f) & =T\left(r, F_{1}\right)+O(1) \\
& \leq \bar{N}\left(r, \frac{1}{F_{1}}\right)+\bar{N}\left(r, \frac{1}{F_{1}-c}\right)+\bar{N}\left(r, F_{1}\right)+S(r, f) \\
& \leq \bar{N}\left(r, \frac{1}{F_{1}}\right)+\bar{N}\left(r, \frac{1}{G_{1}}\right)+\bar{N}\left(r, F_{1}\right)+S(r, f) \\
& \leq(2 n+3) T(r, f)+S(r, f),
\end{aligned}
$$

which means $m-n-2<0$, a contradiction.

Therefore, $c=0$, and by Lemma 2.4 , we have $f \equiv g$.

\section{Proofs of Theorems 1.4 and $\mathbf{1 . 6}$}

The proofs of Theorems 1.4 and 1.6 are similar to that of Theorem 1.2. Noting that

$$
\begin{aligned}
\bar{N}_{(3}\left(r, \frac{1}{F-1}\right) & \leq \frac{1}{2} N\left(r, \frac{F}{F^{\prime}}\right) \leq \frac{1}{2} N\left(r, \frac{F^{\prime}}{F}\right)+S(r, f) \\
& \leq \frac{1}{2} \bar{N}(r, F)+\frac{1}{2} \bar{N}\left(r, \frac{1}{F}\right)+S(r, f) \\
& \leq \frac{1}{2}\left(\bar{N}(r, f)+\bar{N}\left(r, \frac{1}{f}\right)+\sum_{i=1}^{n} N\left(r, \frac{1}{f-\omega_{i}}\right)+N\left(r, \frac{1}{f^{\prime}}\right)\right)+S(r, f) \\
& \leq\left(2+\frac{n}{2}\right) T(r, f)+S(r, f)
\end{aligned}
$$


and

$$
\begin{aligned}
\bar{N}_{(2}\left(r, \frac{1}{F-1}\right) & \leq N\left(r, \frac{F}{F^{\prime}}\right) \leq N\left(r, \frac{F^{\prime}}{F}\right)+S(r, f) \\
& \leq \bar{N}(r, F)+\bar{N}\left(r, \frac{1}{F}\right)+S(r, f) \\
& \leq \bar{N}(r, f)+\bar{N}\left(r, \frac{1}{f}\right)+\sum_{i=1}^{n} N\left(r, \frac{1}{f-\omega_{i}}\right)+N\left(r, \frac{1}{f^{\prime}}\right)+S(r, f) \\
& \leq(4+n) T(r, f)+S(r, f),
\end{aligned}
$$

we could obtain the conclusions of Theorems 1.4 and 1.6 analogous to Theorem 1.2 .

\section{Proofs of Theorems 1.3, 1.5, and 1.7}

Since the terms $N(r, f)$ and $N(r, g)$ equal to $O(1)$ now, analogous to the proofs of Theorems 1.2, 1.4 and 1.6, we could get the conclusions of Theorems 1.3, 1.5 and 1.7 .

Concluding Remark. From the conclusion of Theorem 1.2, we could say that the non-linear differential equations about $f$ 's

$$
f^{m}\left(f^{n}-1\right) f^{\prime}-1=\gamma(z)
$$

may have a sole meromorphic solution for at most one $\gamma(z) \in \Gamma$ with the assumptions that $m>n+10, n \geq 2$ and $(m+1, n)=1$, where $\gamma(z)$ is a meromorphic function, and $\Gamma$ is a family of meromorphic functions such that any two elements $\gamma_{1}(z), \gamma_{2}(z) \in \Gamma$ satisfy the condition that $E_{3)}\left(0, \gamma_{1}\right)=E_{3)}\left(0, \gamma_{2}\right)$. In particular, if $\Gamma$ is a family of entire functions such that its elements have the same property as above, then the non-linear differential equations may have a sole entire solution for at most one $\gamma(z) \in \Gamma$ provided that $m>n+5$ by the conclusion of Theorem 1.3. Similar discussions could be done about the solvability of the non-linear differential equations above by the conclusions of Theorems 1.4-1.7 and we omit the details here.

Acknowledgement. The authors are indebted to Professor Hong-Xun Yi and the referee for reading through the paper and giving lots of valuable suggestions.

\section{References}

[1] J. Clunie, On integral and meromorphic functions, J. London Math. Soc. 37 (1962), $17-27$.

[2] _ On a result of Hayman, J. London Math. Soc. 42 (1967), 389-392.

[3] M. L. Fang and W. Hong, A unicity theorem for entire functions concerning differential polynomials, Indian J. Pure Appl. Math. 32 (2001), no. 9, 1343-1348.

[4] W. K. Hayman, Meromorphic Functions, Clarendon Press, Oxford, 1964.

[5] _ Research Problems in Function Theory, The Athlone Press University of London, London 1967.

[6] Picard values of meromorphic functions and their derivatives, Ann. of Math. (2) $\mathbf{7 0}$ (1959), 9-42. 
[7] P. C. Hu, P. Li, and C. C. Yang, Unicity of Meromorphic Mappings, Advances in Complex Analysis and its Applications, 1. Kluwer Academic Publishers, Dordrecht, 2003.

[8] W. C. Lin and H. X. Yi, Uniqueness theorems for meromorphic function, Indian J. Pure Appl. Math. 35 (2004), no. 2, 121-132.

[9] C. C. Yang, On deficiencies of differential polynomials. II, Math. Z. 125 (1972), 107112.

[10] C. C. Yang and X. H. Hua, Uniqueness and value-sharing of meromorphic functions, Ann. Acad. Sci. Fenn. Math. 22 (1997), no. 2, 395-406.

[11] _ Unique polynomials of entire and meromorphic functions, Mat. Fiz. Anal. Geom. 4 (1997), no. 3, 391-398.

[12] C. C. Yang and H. X. Yi, Uniqueness Theory of Meromorphic Functions, Mathematics and its Applications, 557. Kluwer Academic Publishers Group, Dordrecht, 2003.

[13] H. X. Yi, Meromorphic functions that share one or two values, Complex Variables Theory Appl. 28 (1995), no. 1, 1-11.

Junfeng Xu

Department of Mathematics

WUYI UNIVERSITY

Jiangmen, Guangdong 529020, P. R. China

E-mail address: xujunf@gmail.com

QI HAN

Department of Mathematics

UNIVERSITY OF HOUSTON

Houston, TX 77204-3476, U. S. A.

E-mail address: kylinhan@uh.edu

JILONG ZHANG

LMiB and Department of Mathematics

BeIHANG University

BeiJing 100083, P. R. China

E-mail address: jilongzhang2007@gmail.com 